\title{
Trajetórias educacionais como evidência da qualidade da educação básica brasileira*
}

\author{
José Francisco Soares ${ }^{\star \star}$ \\ Maria Teresa Gonzaga Alves ${ }^{\star \star *}$ \\ José Aguinaldo Fonseca ${ }^{\star \star \star \star}$
}

Segundo a Constituição Federal brasileira, o direito à educação significa a garantia do acesso à escola, onde a população deve permanecer durante o tempo legalmente determinado, a fim de adquirir os conhecimentos e habilidades necessários para a vida adulta plena. Este artigo tem como foco a análise da permanência dos estudantes na escola, condição essencial para a aprendizagem e representada por suas trajetórias educacionais. Dados longitudinais do Censo Escolar, de 2007 a 2015, foram utilizados para analisar as trajetórias educacionais classificadas em três tipos: regular; com poucas irregularidades; e com muitas irregularidades. Os resultados mostram que alguns grupos sociais são muito mais propensos a ter uma trajetória regular e outros a ter repetência ou a ficar fora da escola em alguns anos. Uma proporção alta de meninos de cor/raça preta tem trajetórias com muitas irregularidades. 0 artigo mostra que o Índice de Desenvolvimento da Educação Básica (Ideb) - o indicador oficial para medir a qualidade da educação nos municípios - pode atingir um valor alto, mesmo que muitos estudantes tenham trajetórias irregulares. Isso evidenciou o efeito da seletividade embutido nesse indicador. Em conclusão, este trabalho sugere a incorporação das trajetórias educacionais no monitoramento da qualidade da educação nos municípios.

Palavras-chaves: Direito à educação. Qualidade da educação. Trajetórias educacionais. Dados longitudinais. Educação básica. Censo Escolar.

\footnotetext{
* 0 presente trabalho foi desenvolvido no âmbito da pesquisa "Trajetórias escolares dos estudantes brasileiros na educação básica e no ensino superior”, do Núcleo de Pesquisa em Desigualdades Escolares (Nupede) da Universidade Federal de Minas Gerais (UFMG), e parcialmente apoiado pelo Conselho Nacional de Desenvolvimento Científico e Tecnológico (CNPq) por meio do projeto interinstitucional "Estratificação da educação básica brasileira: uma abordagem multidimensional" (Processo n. 440172/2017-9).

${ }^{\star \star}$ Universidade Federal de Minas Gerais (UFMG), Belo Horizonte-MG, Brasil (francisco.soares.ufmg@gmail.com; https:// orcid.org/0000-0002-1630-4805).

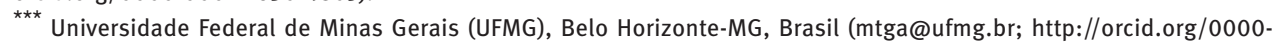
0001-5820-4311).

**** Pontifícia Universidade Católica de Minas Gerais (PUC Minas), Belo Horizonte-MG, Brasil (jafonsecca@gmail.com; https://orcid.org/0000-0002-5894-7008).
} 


\section{Introdução}

0 artigo da Constituição Federal que garante o direito à educação estabelece a sua definição operacional - o “[...] pleno desenvolvimento da pessoa, seu preparo para o exercício da cidadania e sua qualificação para o trabalho" - e as responsabilidades para que esse direito seja garantido - "a educação, direito de todos e dever do Estado e da família, será promovida e incentivada com a colaboração da sociedade” (BRASIL, 1988, art. 205). Cada uma dessas dimensões deve ser monitorada com metodologias próprias.

A oferta de escolas em condições adequadas de funcionamento é a principal evidência do cumprimento do dever do Estado. A recente aprovação do novo Fundo de Manutenção e Desenvolvimento da Educação Básica e de Valorização dos Profissionais da Educação (Fundeb) trouxe a questão do financiamento para o centro do debate educacional, conforme discutido por Callegari (2020). Compete também ao Estado criar as normas educacionais, executadas no âmbito dos entes federados.

Este trabalho se concentra na dimensão operacional do direito à educação por meio da análise da permanência dos estudantes na escola, condição essencial para o aprendizado. No entanto, aceitamos a formulação de "resultados abrangentes", de Sen (2009), e assumimos que os resultados devem ser considerados conjuntamente com os processos e as condições que possibilitaram a sua ocorrência. Assim, o foco na análise de resultados não significa uma secundarização dos insumos e processos.

Atualmente, o monitoramento dos resultados educacionais é realizado por meio de indicadores de acesso, permanência e aprendizado. 0 Censo Demográfico e a Pesquisa Nacional por Amostra de Domicílio Contínua (PNAD Contínua), realizados pelo Instituto Brasileiro de Geografia e Estatística (IBGE), produzem os dados para o monitoramento do acesso à educação (RIANI; GOLGHER, 2004). Sabemos, por meio da PNAD Contínua 2018, que 0 atendimento escolar está quase universalizado nas faixas etárias obrigatórias por lei, uma vez que $98,1 \%$ das crianças de seis a 14 anos, 93,8\% na faixa etária de quatro a cinco anos e 92,9\% entre os jovens de 15 a 17 anos frequentam a escola (INEP, 2020). Mas o percentual de jovens de 16 anos com o ensino fundamental completo ainda é baixo - somente $75,8 \%$, segundo essa mesma pesquisa -, sendo que quase um quarto ficou para trás. Cabe ressaltar que concluir o ensino fundamental aos 16 anos é uma idade acima da teoricamente ideal para essa etapa. Isso é uma evidência de problema nas trajetórias educacionais.

O Instituto Nacional de Estudos e Pesquisas Educacionais Anísio Teixeira (Inep) é responsável pelo monitoramento da permanência e do aprendizado. 0 Censo Escolar coleta informações anuais sobre as condições da oferta e as matrículas, utilizadas para o cálculo das taxas de rendimento e de transição escolar (RIGOTTI; CERQUEIRA, 2004).

Em um sistema educacional eficaz, todos os estudantes da mesma coorte de nascimento seriam matriculados na mesma idade em uma escola de educação básica e permaneceriam no sistema até a conclusão desse nível de ensino na idade fixada na lei. Esse ideal 
está longe de ser alcançado. Em 2017, 9\% dos alunos do ensino fundamental e 16,9\% do ensino médio foram reprovados. Na transição entre séries, de 2017 para 2018, 11,5\% e $20,5 \%$, respectivamente nesses níveis de ensino, não foram matriculados na série seguinte. As taxas de distorção idade-série, em 2018 , atingiram $17,2 \%$ no ensino fundamental e $28,2 \%$ no médio. ${ }^{1}$ Esses resultados indicam que as disfunções no rendimento escolar são potencializadas ao longo da trajetória educacional.

Os resultados do aprendizado são monitorados pelo Sistema de Avaliação da Educação Básica (Saeb). ${ }^{2}$ Nessa dimensão, o Brasil tem avançado, mas permanece abaixo do nível considerado adequado (SOARES; DELGADO, 2016; ALVES, 2020). Em 2017, considerando somente os resultados das escolas públicas, o melhor desempenho foi alcançado no 5 응 ano, em língua portuguesa, com $56 \%$ dos estudantes nos níveis proficiente e avançado. Para um sistema de ensino ser considerado de qualidade, pelo menos $70 \%$ deveriam estar nesses níveis. ${ }^{3}$

De forma global, a qualidade da educação é monitorada pelo Índice de Desenvolvimento da Educação Básica (Ideb), calculado pelo produto do rendimento escolar (média das taxas de aprovação nas etapas de ensino) com o aprendizado (média das proficiências do Saeb) (BRASIL, 2007). O Ideb possui metas de melhoria bianuais para o Brasil, os estados, os municípios e as escolas. 0 indicador tem como pressuposto uma "taxa de troca" entre a proficiência esperada dos estudantes ao final de uma etapa de ensino e o tempo médio de duração para sua conclusão, de forma que os sistemas de ensino devem buscar melhorar o aprendizado sem aumentar as reprovações (FERNANDES, 2007).

O Ideb se tornou um dos principais componentes na formulação e monitoramento das políticas educacionais (ARAÚJO; CODES; UDERMAN, 2019; CAVALCANTI; OLIVEIRA, 2019). No entanto, a literatura aponta algumas limitações relacionadas à sua métrica, fidedignidade, viabilidade de suas metas e associação de seus resultados ao nível socioeconômico das escolas (ALVES; SOARES, 2013; SOARES; XAVIER, 2013; MATOS; RODRIGUES, 2016; PONTES; SOARES, 2016; TRAVITZKI, 2020).

Um tema ausente nessa discussão é a seletividade embutida no cálculo do Ideb. A evasão, o abandono ou a reprovação em anos-calendários anteriores àquele em que o Ideb é calculado não o afetam. Dito de outro modo, os estudantes que saem da trajetória regular não impactam no valor do Ideb. Isso limita sua utilidade para a sugestão e o monitoramento do atendimento do direito à educação, que por definição é para todos. Além disso, o desempenho no Saeb não mede o aprendizado de uma coorte, mas sim daqueles que atingiram

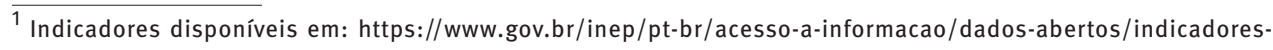
educacionais. Acesso em: 15 fev. 2021.

2 Informações sobre o Saeb disponíveis em: https://www.gov.br/inep/pt-br/areas-de-atuacao/avaliacao-e-exameseducacionais/saeb. Acesso em: 10 fev. 2021.

${ }^{3}$ Os níveis de aprendizado foram propostos por Soares (2009), que divide a escala do Saeb em quatro: insuficiente, básico, proficiente e avançado. Os pontos de corte na escala variam por etapa de ensino e área avaliada. Resultados do Saeb por nível de proficiência podem ser consultados em: https://www.qedu.org.br/brasil/proficiencia. Acesso em: 10 fev. 2021.
} 
os anos finais das etapas escolares. Uma parte dos estudantes que ingressaram na escola aos seis/sete anos de idade fica para trás em função de reprovação, abandono ou evasão.

Sem desconsiderar a importância do Ideb para o monitoramento da qualidade da educação, este artigo tem como foco a trajetória educacional, que é um indicador diferente das taxas de transição entre séries divulgadas pelo Inep. A trajetória regular de um estudante que permaneceu na escola é um resultado educacional que antecede ao desempenho e, portanto, é uma expressão essencial do atendimento do direito à educação.

A análise das trajetórias educacionais exige dados longitudinais que acompanham uma coorte. Isso se tornou possível a partir de 2007, quando o Censo Escolar passou a coletar dados de cada estudante. 0 principal objetivo deste artigo é, portanto, descrever as trajetórias de uma coorte de estudantes que ingressaram na educação básica nesse ano.

A seção a seguir apresenta os conceitos utilizados e a metodologia para tratamento dos dados. Os resultados estão organizados em três subseções - trajetórias educacionais, resultados municipais e probabilidade de exclusão da trajetória regular. Nas considerações finais é discutida a importância de políticas públicas que incidam sobre a permanência dos estudantes na escola.

\section{Trajetórias educacionais}

\section{Conceitos}

A cada ano, o sistema escolar registra a matrícula de cada estudante em uma etapa de ensino e em uma escola. No fim do ano, a esse estudante é atribuída uma situação aprovado, reprovado ou abandono - que expressa o rendimento escolar.

O estudante é aprovado quando concluiu o ano escolar com sucesso e está apto a se matricular na próxima etapa no ano seguinte; ele é reprovado quando não obteve êxito ao fim do ano letivo por desempenho insuficiente e/ou infrequência e, portanto, não está apto a se matricular na próxima etapa no ano seguinte. Alguns estudantes deixam de frequentar a escola durante o ano letivo e por isso não têm a informação de rendimento. São os que abandonam a escola.

A aprovação em todos os anos, que garante a progressão contínua, é a situação desejada. Mas essa não é a realidade de muitos estudantes. Aqueles que abandonam a escola podem não aparecer por ocasião de nova matrícula no ano seguinte, situação classificada como evasão. Isso pode ocorrer mesmo com estudantes que tiveram algum rendimento no ano anterior. Por isso, para construir a trajetória dos estudantes, é preciso considerar o seu fluxo escolar, conceito diferente do rendimento.

Pelos indicadores de fluxo escolar, o estudante pode ser classificado tipicamente em uma dessas três situações: promovido, quando ele se matricula no ano/etapa seguinte em relação ao ano anterior; repetente, quando ele se matricula no mesmo ano/etapa do ano anterior; e evadido quando ele não se matricula. Além dessas, há a 
possibilidade de migração para a modalidade de Educação de Jovens e Adultos (EJA), no caso de estudantes que não concluíram o ensino fundamental ou o médio com até dois anos acima da idade ideal.

Um estudante teve sucesso escolar se foi promovido, e insucesso se não foi promovido ou evadiu do sistema. As experiências de trajetórias educacionais com irregularidades em função de reprovação, abandono ou evasão - tendem a produzir efeitos cumulativos negativos no curso de vida (RIOS-NETO; GUIMARÃES, 2010; TINGLE; SCHOENEBERGER; ALGOZZINE, 2012; ANDREW, 2014; FERRÃO; COSTA; MATOS, 2017; ALEXIADOU; HELGØY; HOMME, 2019; ALVES; FERRÃO, 2019; GIANO; WILLIAMS; BECNEL, 2021). Dessa forma, definimos uma trajetória como regular se o estudante tem sucesso em todos os anos/ calendário; ou como irregular se em algum ano/calendário há registro de repetência, abandono ou evasão.

O Censo Escolar produz há muitos anos informações para a análise das taxas de rendimento (aprovação, reprovação e abandono) e de transição entre séries. Ribeiro (1991) cunhou o termo "pedagogia da repetência", o que ensejou muitos estudos posteriores e a própria mudança na metodologia do Censo. Trata-se de uma contribuição muito importante, mas que, ao não considerar a trajetória de cada estudante, deixa de caracterizar com precisão quem são os excluídos. A caracterização das trajetórias educacionais exige dados que só se tornaram disponíveis recentemente no Brasil.

\section{Dados}

A partir de 2007, o Censo Escolar passou a ser feito para cada estudante. 0 Inep atribui a todos os alunos matriculados em qualquer escola de educação básica um código de identificação único, usado no preenchimento das informações de cada estudante no Censo Escolar anualmente. Por meio desse código, é possível acompanhar as trajetórias dos alunos ao longo dos anos.

Os dados do Censo Escolar são coletados para funções administrativas, mas lentamente foram ganhando a validade necessária para serem usados em pesquisas. Com a ligação dos IDs (código Inep) entre edições do Censo Escolar, é possível construir uma base de bancos longitudinais, como fizeram Oliveira e Menezes Filho (2018) e Paula e Alves (2019), ambos com informações das matrículas em Belo Horizonte, MG.

Mas uma grande contribuição para o uso desses dados longitudinais foi fornecida pelo esforço do Inep de verificação da consistência desses dados. Em particular, muitas duplicações que ocorreram na implementação do Censo Escolar com informações individuais dos estudantes foram eliminadas. Os dados utilizados neste trabalho foram extraídos da base longitudinal dos estudantes preparada pelo Inep. ${ }^{4}$ Eles diferem, portanto, das informações anteriormente publicadas.

\footnotetext{
${ }^{4}$ Os dados foram obtidos junto ao Cedeplar - Centro de Desenvolvimento e Planejamento Regional da Faculdade de Ciências Econômicas da Universidade Federal de Minas Gerais (UFMG), que, por meio de um acordo de cooperação entre o Inep e a UFMG, fornece $o$ acesso às bases de dados do Inep para fins de pesquisa.
} 
Essa base de dados longitudinal foi utilizada em pesquisas recentes. Rigotti e Hadad (2018), em uma investigação sobre a relação entre migrações e trajetórias de 3.589 .462 estudantes nascidos entre 2000 e 2001 , constataram que apenas $30 \%$ desses estudantes apresentavam trajetória regular de 2007 a 2015. Em um estudo exploratório sobre o perfil de 4.000.414 alunos com registro de matrícula no 1 ํano do ensino fundamental em 2007, Alves e Soares (2019) encontraram 38\% com trajetória regular no mesmo período. Os diferentes recortes nessa base de dados levaram a conclusões ligeiramente distintas, mas ambos mostraram um resultado educacional negativo no que diz respeito à permanência dos estudantes com a sua coorte.

Em relação aos estudos prévios, este artigo inova na forma de tratamento dos dados. Buscamos minimizar algumas inconsistências decorrentes de variações na idade de ingresso nos diferentes sistemas de ensino, das reclassificações dos estudantes e possíveis erros de preenchimento dos dados administrativos.

Inicialmente, selecionamos os registros administrativos de 2007 a 2015 da coorte de estudantes nascidos entre $1^{\circ}$ de julho de 1999 e 30 de junho de 2000 . 0 período coberto pelas datas de nascimento reflete a prática vigente em 2007 de matricular no primeiro ano do ensino fundamental os estudantes que nasceram no primeiro semestre do ano em que completam seis anos. Isso foi mudado depois, quando ficou estabelecida a data de 31 de março para o corte etário.

A coorte selecionada é constituída por 3.318.649 estudantes. Como mostra os dados da Tabela 1, em 2007, a maioria estava matriculada no $1^{0}$ ano do ensino fundamental de nove anos $(41,4 \%)$. Mas havia muitos estudantes no $2^{\circ}$ ano $(25,2 \%)$, na educação infantil $(23,7 \%)$ e alguns que só foram registrados na base de dados nos anos seguintes, portanto, sem matrícula (8,8\%). Em 2007, o ensino fundamental estava em processo de ampliação de oito para nove anos de duração, com a obrigatoriedade da matrícula de crianças a partir dos seis anos de idade, mas foi estabelecido um prazo de implantação até 2010 (BRASIL, 2006). Muitas crianças com seis anos completos ainda estavam na educação infantil ou sem matrículas em 2007.

A verificação desses dados mostrou que há três categorias de estudantes que não devem ser usadas nas análises. A primeira é constituída pelos falecidos; a segunda é composta por estudantes com matrículas em anos escolares incompatíveis com sua idade; e a terceira é formada por alunos com oito anos, mas com registro de matrícula apenas em 2007. Como se tratava do primeiro ano com a nova forma de coleta de dados no Censo, erros podem ter ocorrido.

Para definir a segunda categoria de exclusão, verificamos a consistência das informações referentes aos estudantes cuja matrícula, em 2007, é referenciada na Tabela 1 como “outras etapas/ano" (0,9\%). Encontramos casos de estudantes cujo ano escolar, em 2007, não era compatível com a data de nascimento. Tomamos o cuidado de separar possíveis erros de preenchimento pela escola da data de nascimento do estudante daqueles que tiveram entrada antecipada no sistema escolar. Também encontramos estudantes que, durante 
a trajetória, "adiantaram" um ano escolar para ajustar a matrícula do ensino fundamental de oito anos para o de nove anos, bem como outras situações decorrentes da mudança na organização do ensino que ocorreu no período acompanhado. Essas informações, tanto para 2007 quanto para os demais anos, foram analisadas e, quando inconsistentes, excluídas.

A terceira categoria resultou da análise da informação sobre o fluxo do estudante entre anos escolares. A variável fluxo escolar possui os seguintes códigos: (1) promovido; (2) repetente; (3) evadido de escola; (4) migração para a Educação de Jovens e Adultos (EJA); (5) nova escola; (6) migração para o regular; (7) falecido; e (9) não se aplica, categoria usada quando a matrícula refere-se a uma modalidade não seriada da educação básica, como a creche, educação infantil e EJA. Considerando a idade dos estudantes da coorte selecionada, aqueles com códigos 4, 6 e 9 foram excluídos por se tratar de claros erros de informação. Importante observar que isso não se aplica ao ano de 2007, quando os estudantes da coorte estavam completando sete anos. Por precaução, resolvemos não excluir os alunos cujo registro indicava que a matrícula em 2007 era na educação infantil.

Ao final, restaram 3.190.109 estudantes, tendo sido excluída da análise apenas uma pequena porcentagem, conforme mostra a Tabela 1. 0 maior percentual de exclusão foi do grupo de estudantes matriculados em outras etapas/ano, mas sob o menor percentual de casos iniciais.

TABELA 1

Total de matrículas para a coorte 1999/2000, casos excluídos e incluídos, segundo etapa/ano escolar Brasil - 2007

\begin{tabular}{|c|c|c|c|c|c|c|}
\hline \multirow[b]{2}{*}{ Etapa/ano } & \multicolumn{2}{|c|}{ Total de matrículas } & \multicolumn{2}{|c|}{ Excluídos } & \multicolumn{2}{|c|}{ Incluídos } \\
\hline & $\mathrm{N}$ & $\begin{array}{c}\text { \% por } \\
\text { etapa/ano }\end{array}$ & $\mathrm{N}$ & $\%$ & $\mathrm{~N}$ & $\%$ \\
\hline Educação infantil & 787.630 & 23,7 & 19.365 & 2,5 & 768.265 & 97,5 \\
\hline 10 ano EF & 1.373 .150 & 41,4 & 29.481 & 2,1 & 1.343 .669 & 97,9 \\
\hline $2^{\circ}$ ano EF & 837.097 & 25,2 & 51.210 & 6,1 & 785.887 & 93,9 \\
\hline Outras etapas/ano & 29.474 & 0,9 & 7.192 & 24,4 & 22.282 & 75,6 \\
\hline Sem matrícula & 291.298 & 8,8 & 21.292 & 7,3 & 270.006 & 92,7 \\
\hline Total & 3.318 .649 & 100,0 & 128.540 & 3,9 & 3.190 .109 & 96,1 \\
\hline
\end{tabular}

Fonte: Dados longitudinais do Censo Escolar (Inep/Cedeplar-UFMG). Elaboração dos autores.

\section{Classificação das trajetórias}

Uma trajetória educacional é definida como a associação entre dois vetores: o primeiro contendo as idades de cada estudante na data do corte etário (30 de junho); e o segundo referente aos anos escolares em que cada estudante se matriculou. 0 Quadro 1 mostra uma trajetória completamente regular para os estudantes que tinham seis anos completos em 30/06/2007. 
QUADRO 1

Trajetória regular

\begin{tabular}{llllllllll}
\hline \multicolumn{1}{c}{ Anos/ calendário } & 2007 & $\mathbf{2 0 0 8}$ & $\mathbf{2 0 0 9}$ & $\mathbf{2 0 1 0}$ & $\mathbf{2 0 1 1}$ & $\mathbf{2 0 1 2}$ & $\mathbf{2 0 1 3}$ & $\mathbf{2 0 1 4}$ & $\mathbf{2 0 1 5}$ \\
\hline Idade & 6 & 7 & 8 & 9 & 10 & 11 & 12 & 13 & 14 \\
Ano escolar de matrícula & 1 & 2 & 3 & 4 & 5 & 6 & 7 & 8 & 9 \\
\hline
\end{tabular}

Desvios dessa trajetória ideal podem acontecer por vários motivos, tais como entrada antecipada ou tardia no sistema escolar, reclassificação, abandono, repetência, evasão e erros de registro. 0 número de perfis de trajetórias observado na coorte de estudantes analisada é muito grande, chegando a 80.009. É necessário agrupá-los em um pequeno número de categorias.

Para isso, definimos cinco critérios de qualidade para as trajetórias, que foram usados para contar as intercorrências presentes em cada trajetória: presença de evasão; número de anos de sucesso escolar; número de anos de atraso escolar; ano escolar atingido em 2015; e presença de repetência.

Esses cinco critérios associados são necessários para captar a qualidade da enorme diversidade de trajetórias encontrada. Para efeito de criação de tipos de trajetória, os dois primeiros critérios geraram até duas intercorrências e os outros uma intercorrência, como explicado a seguir.

Para calcular o primeiro critério - a presença de evasão -, verificamos se o estudante foi ou não matriculado em cada ano/calendário, situação registrada em uma variável construída a partir da existência de um código de escola no registro do estudante em cada ano. Os estudantes sem essa informação foram considerados como fora da escola no respectivo ano. Essa informação foi discretizada: os estudantes sem evasão receberam o valor 0 , os com um ano fora da escola o valor 1 e os outros o valor 2.

O número de anos de sucesso escolar - segundo critério - é entendido como a matrícula em ano escolar maior do que o ano escolar da matrícula anterior. Inicialmente, o fluxo dos estudantes foi categorizado em sucesso ou insucesso. Se o estudante não esteve fora da escola e se seu fluxo em cada um dos anos letivos, entre 2007 e 2015, foi de "promovido", então esse estudante foi alocado na categoria 1 da variável sucesso escolar no respectivo ano. Se, por outro lado, esteve matriculado, mas não foi promovido, foi incluído na categoria 0 . Além dessas duas situações, os estudantes podem ser classificados na categoria 1 em alguns casos especiais, envolvendo sua matrícula na educação infantil e as condições especiais em 2007. Por exemplo, o estudante sem informação em 2007, mas com oito progressões nos anos seguintes, foi alocado em uma situação de trajetória regular. Ao final, os estudantes com oito ou nove anos de sucesso receberam valor 0 , os com sete e seis anos o valor 1 e os outros o valor 2 .

Pelo terceiro critério, calculamos o valor do atraso escolar como a diferença entre a idade dos estudantes no ano/calendário e o ano escolar em que ocorreu a matrícula. Tomamos o maior valor do atraso como a terceira medida de qualidade da trajetória. A informação referente a 2007 não foi considerada porque, nesse ano, a mudança entre o fundamental de oito anos e o de nove anos ainda gerava muitas inconsistências. A informação sobre 
o atraso foi dicotomizada. Os estudantes sem atraso receberam o valor 0 e aqueles com algum atraso o valor 1 .

O quarto critério contempla o ano escolar alcançado ao fim do período de acompanhamento. Os estudantes dessa coorte deveriam estar no 9 으 ano do ensino fundamental. As escolas que ainda mantinham o ensino fundamental de oito anos tiveram suas informações expressas na notação do ensino de nove anos para o tratamento uniforme dos anos escolares.

0 quinto critério considera a existência de repetência, definida como a matrícula no ano/calendário imediatamente posterior ao ano de uma matrícula, no mesmo ano escolar.

A soma dos cinco critérios varia entre 0 e 7 . As ocorrências com 0 ou 1 foram alocadas no tipo 1 (trajetória regular); as com 2 ou 3 no tipo 2 (trajetória com poucas irregularidades); e as outras no tipo 3 (trajetória com grandes irregularidades).

\section{Características dos estudantes e escolas}

O Censo Escolar coleta a informação sobre sexo e cor/raça dos estudantes. No entanto, há muitos dados ausentes para a variável cor/raça. Para cada estudante, foi registrada a cor/raça prevalente nos anos.

O índice de nível socioeconômico (NSE) das escolas foi calculado com dados das avaliações educacionais do Inep com a mesma metodologia descrita por Alves, Soares e Xavier (2014). Para este estudo, o índice foi estendido para as outras escolas que não possuem dados das avaliações educacionais. Para isso, utilizamos a informação sobre o percentual de estudantes de famílias beneficiárias do programa Bolsa Família por escola e o Índice de Desenvolvimento Humano-Renda (IDH-Renda) do município de $2010 .{ }^{5}$ Cada estudante foi alocado na categoria da média do NSE da escola em que foi matriculado, ponderado pelo número de presenças do estudante no período, isto é, excluindo, no cálculo da média, os anos sem matrícula em qualquer escola.

Em cada ano escolar, o estudante está registrado em uma escola. Entre 2007 e 2015, ele pode permanecer na mesma escola, mas também pode mudar de escola uma ou mais vezes. Ao ser transferido, o estudante pode trocar de dependência administrativa, mudar de município, de estado e região. Mais de $90 \%$ dos estudantes têm nove ou oito registros de matrículas na mesma região. Já na dependência administrativa da escola, há mais variações, principalmente envolvendo mudanças dentro da rede pública, entre escolas municipais e estaduais, uma vez que $77 \%$ dos estudantes têm oito ou nove registros em uma escola municipal ou estadual no período.

Analisamos tipos de trajetória por região do país e a dependência administrativa da escola em que o estudante permaneceu mais tempo. Mas, para lidar com as mudanças, criamos uma variável que registra a região e/ou dependência administrativa a que o

\footnotetext{
${ }^{5}$ Os autores agradecem o apoio de Tiago Falcão Silva, da Secretaria Nacional de Renda de Cidadania, que os orientou para obter a autorização de uso desses dados. O IDH-Renda dos municípios é calculado com dados do Censo Demográfico. Disponível em: https://www.ipea.gov.br/portal/index.php?option=com_content\&id=19153.
} 
estudante esteve vinculado o maior número de vezes entre 2007 e 2015 . Encontramos menos de $2 \%$ de empates, quando o estudante foi matriculado em duas ou mais regiões ou dependências administrativas o mesmo número de anos escolares. Nesses casos, vinculamos aleatoriamente o estudante a uma das duas ou três categorias com frequência igual.

\section{Resultados}

\section{Trajetórias educacionais dos estudantes}

A distribuição dos estudantes segundo os três tipos de trajetórias educacionais encontra-se na Tabela 2. De 2007 a 2015, 53,1\% dos estudantes brasileiros foram classificados no tipo 1 , de trajetória regular $(\mathrm{R}), 16,5 \%$ no tipo 2, trajetória com poucas irregularidades (PI), e 30,4\% no tipo 3, com grandes irregularidades (GI). Há um nítido gradiente de qualidade da educação pelo prisma da permanência dos alunos, sendo o tipo 1 indicação de uma trajetória mais adequada. Esse resultado evidencia uma profunda disfuncionalidade do sistema educacional brasileiro. 0 número de estudantes com trajetórias irregulares é alto, o que acarreta grandes prejuízos sociais e pessoais.

TABELA 2

Distribuição dos estudantes selecionados da coorte 1999/2000, segundo tipos de trajetória educacionais

Brasil - 2007-2015

\begin{tabular}{lrr}
\hline \multicolumn{1}{c}{ Tipos de trajetória } & N & \% \\
\hline 1 - Regular (R) & 1.693 .919 & 53,1 \\
2- Poucas irregularidades (PI) & 527.045 & 16,5 \\
3 - Grandes irregularidades (GI) & 969.145 & 30,4 \\
Total & 3.190 .109 & 100,0 \\
\hline
\end{tabular}

Fonte: Dados longitudinais do Censo Escolar (Inep/Cedeplar-UFMG). Elaboração dos autores.

Cabe mencionar que o percentual de estudantes com trajetórias regulares é maior do que os registrados em estudos prévios (RIGOTTI; HADAD, 2018; ALVES; SOARES, 2019). As divergências se explicam, entretanto, pelas diferenças na seleção dos dados, as exclusões de casos inconsistentes e a adoção de múltiplos critérios para definir as trajetórias empregadas neste artigo.

Considerando a região onde o estudante permaneceu mais tempo, os maiores percentuais de trajetórias regulares estão nas regiões Sudeste, Centro-Oeste e Sul. No Norte e Nordeste, os percentuais de trajetórias com muitas irregularidades são mais altos do que aqueles referentes às trajetórias regulares.

Em relação às características dos estudantes, observa-se, na Tabela 4, que há 12,4\% a mais de meninas com trajetórias regulares em relação aos meninos. Considerando que existem menos meninas na coorte analisada (49,2\% vs. 50,8\%), esse resultado revela que há um filtro de seletividade para os meninos no sistema escolar. Eles são 
reprovados e abandonam mais e, em consequência, tornam-se minoria na conclusão do ensino fundamental.

TABELA 3

Distribuição dos estudantes selecionados da coorte 1999/2000, por tipo de trajetória, segundo regiões Brasil - 2007-2015

\begin{tabular}{lccc} 
& & & Em porcentagem \\
\hline \multicolumn{1}{c}{ Regiões } & Tipo 1- R & Tipo 2 - PI & Tipo 3 - GI \\
\hline Brasil & 53,1 & 16,5 & 30,4 \\
Norte & 39,7 & 14,6 & 45,8 \\
Nordeste & 40,0 & 16,6 & 43,4 \\
Sudeste & 65,9 & 15,1 & 19,0 \\
Sul & 54,0 & 20,1 & 25,9 \\
Centro-Oeste & 55,3 & 20,1 & 24,6 \\
\hline
\end{tabular}

Fonte: Dados longitudinais do Censo Escolar (Inep/Cedeplar-UFMG). Elaboração dos autores

TABELA 4

Distribuição dos estudantes selecionados da coorte 1999/2000, por tipo de trajetória, segundo sexo Brasil - 2007-2015

\begin{tabular}{lcccc} 
& & & Em porcentagem \\
\hline Sexo & Tipo 1 - R & Tipo 2 - PI & Tipo 3 - GI \\
\hline Homens & 47,0 & 16,5 & 36,4 \\
Mulheres & 59,4 & 16,5 & 24,1 \\
\hline
\end{tabular}

Fonte: Dados longitudinais do Censo Escolar (Inep/Cedeplar-UFMG). Elaboração dos autores.

A Tabela 5 apresenta os resultados por cor/raça do estudante. Apesar de $31 \%$ de "não declaração", os resultados são consistentes. A maioria dos alunos brancos $(64,9 \%)$ tem trajetória regular (tipo 1), enquanto os menores percentuais são dos alunos indígenas (somente $25,1 \%$ ) e pretos ( $42,5 \%$ ). Os estudantes indígenas registram a maior frequência de anos fora da escola. Mas, por ser uma categoria muito pequena ( $0,5 \%$ dos estudantes), geralmente matriculada em escolas próprias, há necessidade de estudos específicos para o entendimento desses padrões. Quanto aos estudantes pretos, as suas desvantagens educacionais são conhecidas e mais uma vez se comprovam neste estudo (SOARES; ALVES, 2003; ALVES; ORTIGÃO; FRANCO, 2007).

TABELA 5

Distribuição dos estudantes selecionados da coorte 1999/2000, por tipo de trajetória, segundo cor/raça Brasil - 2007-2015

Em porcentagem

\begin{tabular}{lccc}
\hline \multicolumn{1}{c}{ Cor/raça } & Tipo 1 - R & Tipo 2 - PI & Tipo 3 - GI \\
\hline Não declarada & 48,8 & 16,7 & 34,6 \\
Branca & 64,9 & 16,1 & 39,1 \\
Preta & 42,5 & 17,7 & 39,8 \\
Parda & 48,2 & 16,7 & 35,0 \\
Amarela & 57,8 & 14,2 & 28,0 \\
Indígena & 25,1 & 13,3 & 61,6 \\
\hline
\end{tabular}

Fonte: Dados longitudinais do Censo Escolar (Inep/Cedeplar-UFMG). Elaboração dos autores. 
A Tabela 6 mostra um gradiente ascendente na proporção de trajetórias regulares (tipo 1) ao se passar do NSE mais baixo (32,5\%) para o NSE mais alto (73,5\%). Entre as escolas de NSE mais baixo, a maioria dos estudantes encontra-se no tipo 3, trajetórias com muitas irregularidades.

TABELA 6

Distribuição dos estudantes selecionados da coorte 1999/2000, por tipo de trajetória, segundo NSE da escola Brasil 2007-2015

Em porcentagem

\begin{tabular}{lccc}
\hline NSE da escola (quintis) & Tipo 1- R & Tipo 2 - PI & Tipo 3 - GI \\
\hline Baixo & 32,5 & 14,9 & 52,7 \\
Médio-baixo & 41,9 & 18,0 & 40,2 \\
Médio & 55,0 & 17,9 & 27,1 \\
Médio-alto & 63,0 & 17,1 & 19,9 \\
Alto & 73,5 & 14,9 & 11,7 \\
\hline
\end{tabular}

Fonte: Dados longitudinais do Censo Escolar (Inep/Cedeplar-UFMG). Elaboração dos autores.

Os dados da Tabela 7 mostram que há muito mais estudantes com trajetórias regulares entre aqueles que fizeram a maior parte da trajetória em escolas privadas $(72,7 \%)$, seguidas pelas federais (63\%) - rede pública com menos de $0,5 \%$ das matrículas nessa etapa. Entre os matriculados em escolas municipais - que reúnem o maior percentual de matrículas - há maior proporção de trajetórias do tipo 3, com grandes irregularidades $(38,1 \%)$.

TABELA 7

Distribuição dos estudantes selecionados da coorte 1999/2000, por tipo de trajetória, segundo dependência administrativa

Brasil - 2007-2015

Em porcentagem

\begin{tabular}{lccc}
\hline $\begin{array}{c}\text { Dependência } \\
\text { administrativa }\end{array}$ & Tipo 1- R & Tipo 2 - PI & Tipo 3 - GI \\
\hline Federal & 63,0 & 20,7 & 16,2 \\
Estadual & 65,6 & 13,6 & 20,8 \\
Municipal & 43,4 & 18,5 & 38,1 \\
Privada & 72,7 & 13,1 & 14,2 \\
\hline
\end{tabular}

Fonte: Dados longitudinais do Censo Escolar (Inep/Cedeplar-UFMG). Elaboração dos autores.

\section{Resultados municipais}

As trajetórias analisadas correspondem às etapas escolares de responsabilidade dos municípios, razão pela qual descrevemos os tipos de trajetória por município. Frequentemente a trajetória de um aluno não foi cumprida em um único município (RIGOTTI; HADAD, 2018). Para obter o número de trajetórias de cada tipo nos municípios, a trajetória de cada estudante foi alocada nos municípios onde estiveram matriculados, ponderada pelos anos escolares frequentados no município. Com isso, produzimos uma medida do sucesso do município em manter seus estudantes na escola. Há uma enorme variação nessa 
medida, como se pode notar no Gráfico 1, que apresenta o histograma da proporção de trajetórias regulares (tipo 1) nos municípios.

GRÁFICO 1

Proporção de estudantes selecionados da coorte 1999/2000 com trajetória regular (tipo 1), por município (ponderada)

Brasil-2007-2015

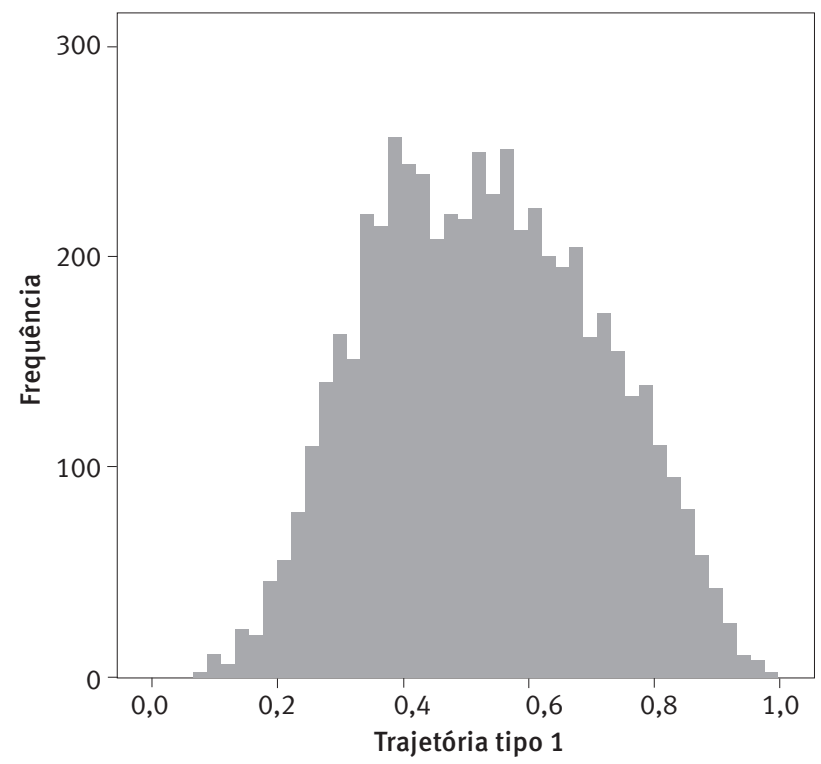

Fonte: Dados longitudinais do Censo Escolar (Inep/Cedeplar-UFMG). Elaboração dos autores.

No entanto, o interesse na descrição do sucesso dos municípios em manter seus estudantes na escola é verificar como essa síntese dialoga com o Ideb. Tomamos o Ideb 2015 referente aos anos finais do ensino fundamental das escolas públicas nos municípios para esta análise. A escala original desse indicador foi categorizada em cinco faixas, conforme Soares e Xavier (2013).

Há 334 municípios sem informação, porque suas escolas não atingiram o número mínimo de alunos avaliados no Saeb. Além disso, em 2015, somente o município de Sebastianópolis do Sul (SP) se classificou na faixa mais alta do Ideb (valor maior que 6,5), que foi um caso atípico naquele ano (outlier), razão pela qual optamos por excluí-lo da análise. A relação do Ideb com os tipos de trajetória é mostrada na Tabela 8. Como esperado, o percentual de estudantes com trajetórias regulares nos municípios é maior nas categorias mais altas do Ideb. Contudo, surpreende-nos que um município seja classificado na categoria mais alta do Ideb com mais de 30\% de trajetórias dos tipos 2 e 3. 
TABELA 8

Distribuição dos estudantes selecionados da coorte 1999/2000, por tipo de trajetória, segundo faixas do Ideb municipal dos anos finais do ensino fundamental

Brasil - 2015

Em porcentagem

\begin{tabular}{llccc}
\hline \multicolumn{2}{c}{ Categorias do Ideb municipal } & Tipo 1 - R & Tipo 2 - PI & Tipo 3 - GI \\
\hline Baixo & $\langle 3,5$ & 35,0 & 15,0 & 49,0 \\
Médio baixo & {$[3,5 ; 4,5]$} & 52,0 & 17,0 & 31,0 \\
Médio & {$[4,5 ; 5,5]$} & 66,0 & 18,0 & 17,0 \\
Médio alto & $>5,5$ & 68,0 & 19,0 & 13,0 \\
\hline
\end{tabular}

Fonte: Dados longitudinais do Censo Escolar (Inep/Cedeplar-UFMG) e Inep. Elaboração dos autores. Nota: Exclui um caso outlier com Ideb maior que 6,5.

A mesma análise foi realizada com dados dos anos iniciais do ensino fundamental, em 2011. Um estudante que ingressou na escola em 2007 e teve uma trajetória regular deveria fazer o Saeb no $5^{0}$ ano em 2011. Os resultados da Tabela 9 são muito parecidos com os da tabela anterior. A diferença importante é que, mesmo entre municípios com valor mais alto do Ideb (acima de 6,5), mais de $30 \%$ dos estudantes estão classificados nos tipos 2 ou 3 de trajetórias.

TABELA 9

Distribuição dos estudantes selecionados da coorte 1999/2000, por tipo de trajetória, segundo faixas do Ideb municipal dos anos iniciais do ensino fundamental

Brasil - 2011

Em porcentagem

\begin{tabular}{lcccc}
\hline \multicolumn{2}{c}{ Categorias do Ideb municipal } & Tipo 1-R & Tipo 2 - PI & Tipo 3 - GI \\
\hline Baixo & $<3,5$ & 33,0 & 15,0 & 53,0 \\
Médio baixo & {$[3,5 ; 4,5]$} & 41,0 & 17,0 & 42,0 \\
Médio & {$[4,5 ; 5,5]$} & 60,0 & 17,0 & 23,0 \\
Médio alto & {$[5,5 ; 6,5]$} & 66,0 & 18,0 & 16,0 \\
Alto & $>6,5$ & 69,0 & 18,0 & 13,0 \\
\hline
\end{tabular}

Fonte: Dados longitudinais do Censo Escolar (Inep/Cedeplar-UFMG) e Inep. Elaboração dos autores.

Essas análises foram repetidas considerando o Ideb dos anos iniciais do ensino fundamental, para 2013 e 2015, e dos anos finais, para 2013 e 2017, a fim de captar as situações atípicas de trajetórias que começaram mais cedo ou atrasadas. Nesses anos, não ocorreram casos atípicos para os anos finais, isto é, a situação de um único município isolado no nível alto. Nessas análises auxiliares, os padrões foram muito semelhantes: 0 percentual de trajetórias do tipo 1 nos municípios com Ideb alto, nos anos iniciais e finais do ensino fundamental, nunca atingiu $70 \%$.

\section{Probabilidades de exclusão da trajetória regular}

As variáveis que classificam os estudantes agem concomitantemente. Assim, é mais informativo estimar, por meio de uma técnica de regressão, as probabilidades de os diferentes perfis de estudantes estarem em cada um dos tipos de trajetória. 
Para isso, ajustamos um modelo de regressão logístico multinominal, no qual a variável resposta é a trajetória categorizada nos três tipos e as variáveis explicativas são: região de residência; dependência administrativa da escola; quartis do NSE da escola; sexo; e cor/ raça do estudante. Usando o valor estimado dos coeficientes das variáveis explicativas, calculamos as probabilidades de cada perfil de estudante estar em um dos três tipos de trajetória. Para sintetizar essas probabilidades, agregamos, pela média, a probabilidade de um estudante ter uma trajetória regular (tipo 1), segundo a combinação de categorias de três variáveis: sexo; cor/raça (branca, parda e preta); e NSE (quartis). A Tabela 10 mostra esse resultado, além do número de estudantes em cada combinação. Não apresentamos as médias das combinações envolvendo estudantes de cor/raça amarela e indígena, considerando o seu pequeno número. 0 resultado completo do modelo está disponível em documento suplementar.

TABELA 10

Médias das probabilidades de o estudante da coorte 1999/2000 estar na trajetória regular e número de estudantes, segundo sexo, cor/raça e NSE da escola

Brasil - 2007-2015

\begin{tabular}{|c|c|c|c|c|}
\hline Sexo & Cor/raça & $\begin{array}{l}\text { Quartil NSE da } \\
\text { escola }\end{array}$ & $\begin{array}{l}\text { Probabilidade de } \\
\text { estar na trajetória } \\
\text { regular }\end{array}$ & N. de estudantes \\
\hline Mulher & Branca & Alto & 0,78 & 200.706 \\
\hline Mulher & Parda & Alto & 0,75 & 68.258 \\
\hline Mulher & Branca & Médio-alto & 0,70 & 148.731 \\
\hline Homem & Branca & Alto & 0,70 & 201.734 \\
\hline Mulher & Preta & Alto & 0,67 & 10.015 \\
\hline Mulher & Parda & Médio-alto & 0,66 & 125.090 \\
\hline Homem & Parda & Alto & 0,66 & 69.311 \\
\hline Mulher & Branca & Médio-baixo & 0,61 & 84.534 \\
\hline Homem & Branca & Médio-alto & 0,59 & 149.980 \\
\hline Mulher & Preta & Médio-alto & 0,57 & 15.520 \\
\hline Homem & Preta & Alto & 0,56 & 10.577 \\
\hline Homem & Parda & Médio-alto & 0,54 & 131.018 \\
\hline Mulher & Parda & Médio-baixo & 0,53 & 165.200 \\
\hline Mulher & Branca & Baixo & 0,50 & 44.171 \\
\hline Homem & Branca & Médio-baixo & 0,48 & 85.609 \\
\hline Homem & Preta & Médio-alto & 0,44 & 17.307 \\
\hline Mulher & Preta & Médio-baixo & 0,43 & 16.113 \\
\hline Mulher & Parda & Baixo & 0,41 & 182.041 \\
\hline Homem & Parda & Médio-baixo & 0,39 & 171.360 \\
\hline Homem & Branca & Baixo & 0,36 & 42.617 \\
\hline Mulher & Preta & Baixo & 0,32 & 11.313 \\
\hline Homem & Preta & Médio-baixo & 0,30 & 17.982 \\
\hline Homem & Parda & Baixo & 0,28 & 188.238 \\
\hline Homem & Preta & Baixo & 0,20 & 13.256 \\
\hline
\end{tabular}

Fonte: Dados longitudinais do Censo Escolar (Inep/Cedeplar-UFMG). Elaboração dos autores.

Nota: Exclui médias das probabilidades envolvendo as categorias de cor/raça amarela e indígena. 
As maiores probabilidades $(>0,70)$ envolvem, com mais frequência, alunas brancas em escolas nos quartis mais altos. Dentre as probabilidades mais baixas $(<0,40)$, predominam os meninos pretos ou pardos, em escolas com NSE baixo ou baixo-médio. Nota-se que a diferença nas probabilidades entre o perfil com maior média - meninas brancas em escolas com NSE alto - e aquele com menor média - meninos pretos em escolas de NSE baixo - é de quase quatro vezes.

0 Gráfico 2 sumariza esses achados em uma apresentação que destaca as diferenças por sexo. No eixo vertical do gráfico está a probabilidade de o estudante ter trajetória tipo 1 e, no eixo horizontal, encontram-se os quartis do NSE da escola. As categorias de cor/raça estão representadas pelos triângulos. As linhas pontilhadas correspondem à média $(0,52)$.

\section{GRÁFICO 2}

Probabilidades de o estudante da coorte 1999/2000 estar na trajetória regular, por NSE, segundo sexo e cor/raça Brasil - 2007-2015

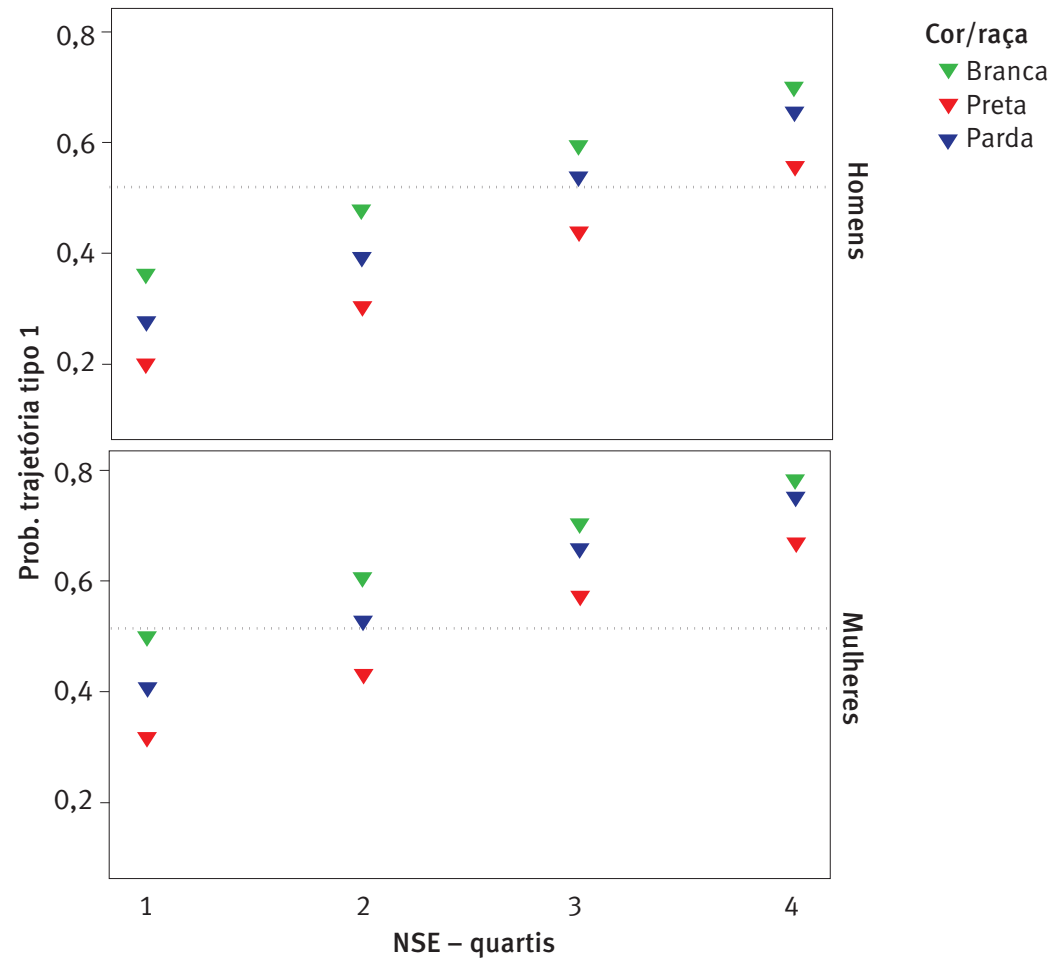

Fonte: Dados longitudinais do Censo Escolar (Inep/Cedeplar-UFMG). Elaboração dos autores.

Três resultados se destacam: o crescimento das probabilidades dos estudantes brancos, pardos e pretos (nessa ordem) com o aumento do NSE, para ambos os sexos; a aproximação das probabilidades de brancos e pardos nos quartis mais altos; e as probabilidades acima da média das meninas brancas e pardas já a partir do segundo quartil de NSE, resultado que os meninos obtêm só a partir do terceiro quartil. 


\section{Considerações finais}

O direito à educação não está plenamente atendido para todas as crianças brasileiras, uma vez que, após o acesso à escola, muitos estudantes têm suas trajetórias interrompidas por uma ou mais barreiras (reprovação, abandono ou evasão). Trajetórias irregulares denotam desigualdades, marca estrutural do país, e se iniciam com a entrada na escola.

Neste artigo, classificamos os estudantes em tipos de trajetórias educacionais e analisamos as probabilidades de determinados perfis terem trajetórias regulares. Os padrões reportados são consistentes e mostram que há grupos sociais mais sujeitos a uma trajetória regular e outros com percurso marcado pelo insucesso. Destacamos as trajetórias dos meninos pretos, com elevado percentual de trajetórias irregulares e chances reduzidas de uma trajetória regular, mesmo quando estudam em escolas de NSE médio-alto. Os resultados indicam que esses estudantes são os mais penalizados pela "pedagogia da repetência" (RIBEIRO, 1991).

Uma constatação nova é que há muitos municípios com valores altos no Ideb, mas que têm também porcentagens elevadas de estudantes com trajetórias irregulares. Tal resultado evidencia o efeito de seletividade embutido nesse indicador. 0 Ideb orienta mal as políticas públicas, por deixar de fora parte importante das dificuldades de muitos estudantes.

Este texto não trata das causas reais do problema. As trajetórias irregulares não são, naturalmente, efeitos de características dos estudantes. As causas reais devem ser buscadas em outras estruturas, como no contexto familiar, nas escolas, que têm condições distintas de funcionamento, de gestão e em suas escolhas pedagógicas (NOGUEIRA, 2005; ALVES et al., 2017; ALVES; XAVIER, 2018). Os resultados não podem, portanto, ser lidos como uma culpabilização dos estudantes. São vítimas, não atores.

O Ideb está no seu último ano e um novo indicador para o monitoramento da qualidade da educação básica deverá substituí-lo. Estudos futuros que investiguem as razões da seletividade embutida no Ideb podem auxiliar no seu aprimoramento. Pelos resultados aqui reportados, recomendamos que o "novo Ideb" considere não somente o aprendizado acumulado no final de cada etapa escolar daqueles estudantes que permaneceram no sistema escolar, mas também a trajetória de cada coorte que ingressa no sistema educacional.

Por fim, a coorte aqui analisada é a primeira para a qual há dados que permitem a construção de trajetórias. Explicitamos os critérios para tratamento dos dados para lidar com possíveis inconsistências nos registros feitos nas escolas. É importante que estudos similares sejam realizados com as coortes posteriores, que terão dados de melhor qualidade, seja pelo aprendizado da nova metodologia, seja pelo desaparecimento do ensino fundamental de oito anos, cuja concomitância com o ensino de nove anos criou limitações nos dados. 


\section{Referências}

ALEXIADOU, N.; HELGØY, I.; HOMME, I. Lost in transition: policies to reduce early school leaving and encourage further studying in Europe. Comparative Education, v. 55, n. 3, p. 297-307, 2019. DOI: 10.1080/03050068.2019.1619327.

ALVES, M. T. G. Caracterização das desigualdades educacionais com dados públicos: desafios para conceituação e operacionalização empírica. Lua Nova: Revista de Cultura e Política, v. 110, p. 189-214, 2020.

ALVES, M. T. G.; FERRÃO, M. E. Uma década da Prova Brasil: evolução do desempenho e da aprovação. Estudos em Avaliação Educacional, v. 30, n. 75, p. 688-720, 2019.

ALVES, M. T. G.; SOARES, J. F. Contexto escolar e indicadores educacionais: condições desiguais para a efetivação de uma política de avaliação educacional. Educação e Pesquisa, v. 39, n. 1, p. 177-194, 2013.

ALVES, M. T. G.; SOARES, J. F. Trajetórias escolares no ensino fundamental: análise longitudinal do Censo Escolar. In: X REUNIÃO DA ABAVE - AVALIAÇÃO EDUCACIONAL NO BRASIL: “O DESAFIO DA QUALIDADE”. Anais [...]. São Paulo: Associação Brasileira de Avaliação Educacional, 2019. Disponivel em: https://www.abave.org.br/xabave/programacao_completa.pdf.

ALVES, M. T. G.; SOARES, J. F.; XAVIER, F. P. Índice socioeconômico das escolas de educação básica brasileiras. Ensaio: Avaliação e Políticas Públicas em Educação, Rio de Janeiro, v. 22, n. 84, p. 671-704, 2014.

ALVES, M. T. G.; XAVIER, F. P. Indicadores multidimensionais para avaliação da infraestrutura escolar: o ensino fundamental. Cadernos de Pesquisa, v. 48, n. 169, p. 708-746, 2018.

ANDREW, $M$. The scarring effects of primary-grade retention? A study of cumulative advantage in the educational career. Social Forces, v. 93, n. 2, p. 653-685, 2014.

ARAÚJO, H. E.; CODES, A.; UDERMAN, L. O Ideb como instrumento de gestão para uma educação de qualidade: a educação brasileira vista pelas lentes do Ideb. Brasília: Ipea, 2019. (Texto para Discussão, 2774).

BRASIL. Presidência da República. Decreto n. 6094, de 24 de abril de 2007. Dispõe sobre a implantação do plano de metas Compromisso Todos Pela Educação. Brasília, 2007.

BRASIL. Presidência da República. Constituição da República Federativa do Brasil de 1988. Brasília, 1988. Disponível em: http://www.planalto.gov.br/ccivil_03/Constituicao/Constituiçao. htm. Acesso em: 19 jan. 2021.

BRASIL. Presidência da República. Lei n. 11.274, de 6 de fevereiro de 2006 - amplia o ensino fundamental para nove anos de duração, com a matrícula de crianças de seis anos de idade e estabelece prazo de implantação, pelos sistemas, até 2010. Brasília, 2006.

CALLEGARI, C. O. Equidade educacional na federação brasileira: o papel das transferências federais aos municípios. Dissertação (Mestrado em Administração Pública) - Fundação Getúlio Vargas, São Paulo, 2020. Disponível em http://bibliotecadigital.fgv.br/dspace/ handle/10438/29010.

CAVALCANTI, C. R.; OLIVEIRA, R. de F. Mecanismos redistributivos na assistência financeira da União no âmbito da educação básica. Educação em Revista, v. 35, 2019.

FERNANDES, R. Índice de Desenvolvimento da Educação Básica (Ideb). Brasília: Inep, 2007. (Série documental. Textos para Discussão, 26). 
FERRÃO, M. E.; COSTA, P. M.; MATOS, D. A. S. The relevance of the school socioeconomic composition and school proportion of repeaters on grade repetition in Brazil: a multilevel logistic model of PISA 2012. Large-scale Assessments in Education, v. 5, n. 1, 2017.

GIANO, Z.; WILLIAMS, A. L.; BECNEL, J. N. Grade retention and school dropout: comparing specific grade levels across childhood and early adolescence. Journal of Early Adolescence, on line fist, 2021. DOI: $10.1177 / 027243162110103322021$.

INEP - Instituto Nacional de Estudos e Pesquisas Educacionais Anísio Teixeira. Relatório do 3o ciclo de monitoramento das metas do Plano Nacional de Educação - 2020 [recurso eletrônico]. Brasília: Inep, 2020.

MATOS, D. A. S.; RODRIGUES, E. C. Indicadores educacionais e contexto escolar: uma análise das metas do Ideb. Estudos em Avaliação Educacional, v. 27, n. 66, p. 662-688, 2016.

NOGUEIRA, M. A. A relação família/escola na contemporaneidade: fenômeno social/interrogações sociológicas. Análise Social, v. XL, n. 176, p. 563-578, 2005.

OLIVEIRA, A. P.; MENEZES FILHO, N. Idade de ingresso escolar, repetência e evasão escolar no Brasil: uma abordagem para estimação de efeitos causais. In: $46^{\circ}$ ENCONTRO NACIONAL DE ECONOMIA. Anais [...]. Rio de Janeiro: Anpec, 2018. Disponível em https://www.anpec.org.br/ encontro/2018/submissao/files_l/i12-016f061e01b865e7419c6f63f8650f07.pdf.

PAULA, T. S.; ALVES, M. T. G. Apontamentos sobre as taxas de fluxo escolar produzidas pelo Inep: um conselho aos navegantes. In:X REUNIÃO DA ABAVE - AVALIAÇÃO EDUCACIONAL NO BRASIL: "O DESAFIO DA QUALIDADE”. Anais [...]. São Paulo: Associação Brasileira de Avaliação Educacional, 2019. Disponível em: https://www.abave.org.br/xabave/programacao_completa.pdf.

PONTES, L. A. F.; SOARES, T. M. As metas escolares do Ideb: uma proposta alternativa de cálculo. Estudos em Avaliação Educacional, v. 27, n. 66, p. 690-715, 2016.

RIANI, J. L.R.; GOLGHER, A. B. Indicadores educacionais confeccionados a partir de bases de dados do IBGE. In: RIOS-NETO, E. L.; RIANI, J. L. R. (org.). Introdução à demografia da educação. Campinas: Associação Brasileira de Estudos Populacionais - Abep, 2004. p. 89-127.

RIBEIRO, S. C. A pedagogia da repetência. Estudos Avançados, São Paulo, v. 5, n. 12, p. 7-21, 1991.

RIGOTTI, J. I. R.; CERQUEIRA, C. A. As bases de dados do Inep e os indicadores educacionais: conceitos e aplicações. In: RIOS-NETO, E. L.; RIANI, J. L. R. (org.). Introdução à demografia da educação. Campinas: Associação Brasileira de Estudos Populacionais - Abep, 2004. p. 73-87.

RIGOTTI, J. I. R.; HADAD, R. M. An analysis of the relationship between internal migration and education in Brazil. Background paper prepared for the 2019 Global Education Monitoring Report. Unesco, 2018. Disponível em: https://unesdoc.unesco.org/ark:/48223/pf0000266085/ PDF/266085eng.pdf.multi.page $=1 \& z 00 \mathrm{~m}=$ auto, $-16,842$.

RIOS-NETO, E. L. G.; GUIMARÃES, R. R. de M. The demography of education in Brazil: inequality of educational opportunities based on Grade Progression Probability (1986-2008). Vienna Yearbook of Population Research, v. 8, p. 283-312, 2010.

SEN, A. K. The idea of justice. Cambridge, MA: Harvard University Press, 2009.

SOARES, J. F. Índice de Desenvolvimento da Educação de São Paulo - Idesp: bases metodológicas. São Paulo em Perspectiva, v. 23, n. 1, p. 29-41, 2009.

SOARES, J. F.; ALVES, M. T. G. Desigualdades raciais no sistema brasileiro de educação básica. Educação e Pesquisa, v. 29, n. 1, p. 147-165, jun. 2003. 
SOARES, J. F.; DELGADO, V. M. S. Medida das desigualdades de aprendizado entre estudantes do ensino fundamental. Estudos em Avaliação Educacional, v. 27, n. 66, p. 754-780, 2016.

SOARES, J. F.; XAVIER, F. P. Pressupostos educacionais e estatísticos do Ideb. Educação \& Sociedade (Cedes), v. 34, n. 124, p. 903-923, 2013.

TINGLE, L. R.; SCHOENEBERGER, J.; ALGOZZINE, B. Does grade retention make a difference? Issues and Ideas, v. 85, n. 5, p. 179-185, 2012.

TRAVITZKI, R. Qual é o grau de incerteza do Ideb e por que isso importa? Ensaio: Avaliação e Políticas Públicas em Educação, v. 28, n. 107, p. 500-520, 2020.

\title{
Sobre os autores
}

José Francisco Soares é pós-doutor em Educação pela University of Michigan, Ann Arbor, e doutor em Estatística pela University of Wisconsin, Madison. Professor emérito da Universidade Federal de Minas Gerais (UFMG). Dedica-se à pesquisa educacional na área de avaliação e da análise dos indicadores síntese, principalmente os referentes às desigualdades nos resultados educacionais.

Maria Teresa Gonzaga Alves é doutora em Educação pela Universidade Federal de Minas Gerais (UFMG) e mestre em Sociologia pela Universidade de São Paulo (USP). Professora associada do Departamento de Ciências Aplicadas à Educação (Decae), da Faculdade de Educação da UFMG. Líder do Núcleo de Pesquisa em Desigualdades Escolares (Nupede).

José Aguinaldo Fonseca é mestre em Estatística Espacial pela Universidade Federal de Minas Gerais (UFMG). Professor da Pontifícia Universidade Católica de Minas Gerais (PUC Minas), nos cursos de Administração, Sistemas de Informação e Engenharias. Consultor na área da Educação, com análise de questões usando a Teoria de Resposta ao Item (TRI).

\section{Endereço para correspondência}

\author{
José Francisco Soares \\ Rua Bianca, 727 \\ 31340-610 - Belo Horizonte-MG, Brasil \\ Maria Teresa Gonzaga Alves \\ Faculdade de Educação, Universidade Federal de Minas Gerais \\ Av. Antônio Carlos, 6627- Pampulha \\ 31270-901 - Belo Horizonte-MG, Brasil \\ José Aguinaldo Fonseca \\ Rua Damas Ribeiro, 600 apto. 12, bloco A \\ 32310-470 - Contagem-MG, Brasil
}

\section{Abstract}

\section{Educational trajectories as evidence of the quality of Brazilian basic education}

According to the Federal Brazilian Constitution, the right to education means that all children had access to a school, and remained there for the legally determined time and, acquired the knowledge and skills necessary for full adult life. This paper focuses on the analysis of students' permanence in school, an essential condition for learning represented here by their educational 
trajectories. The School Census longitudinal data, from 2007 to 2015 were used to analyze the student's educational trajectories classified into three types: regular; with few irregularities; and with many irregularities. The results show that some social groups are much more prone to having a regular trajectory, and others to having grade repetition or being out of school after few years. A large proportion of black boys have trajectories with many irregularities. In addition, this paper shows that the Basic Education Development Index (IDEB, acronym in Portuguese) - the official index for measuring the quality of education in the municipalities - can reach a high value even if many students have irregular trajectories. This evidenced the selectivity effect embedded in this indicator. This paper suggests the incorporation of educational trajectories into the indicator to monitor the quality of education in Brazilian municipalities.

Keywords: Right to education. Quality of education. Educational trajectories. Longitudinal data. Basic education. School census.

\section{Resumen}

Trayectorias educativas como evidencia de la calidad de la educación básica brasileña

De acuerdo con la Constitución Federal brasileña, el derecho a la educación significa la garantía de acceso a la escuela, donde los estudiantes deben permanecer por el tiempo legalmente determinado para adquirir los conocimientos y habilidades necesarios para la vida adulta plena. Este artículo se centra en el análisis de la permanencia de los estudiantes en la escuela, condición esencial para el aprendizaje, y representada por sus trayectorias educativas. Datos longitudinales de los censos escolares desde 2007 hasta 2015 se utilizaron para estudiar las trayectorias educativas del alumno, que se clasificaron en tres tipos: regular, con pocas irregularidades, con muchas irregularidades. Los resultados muestran que algunos grupos sociales son mucho más propensos a tener una trayectoria regular y otros a repetir el grado o quedar fuera de la escuela por algunos años. Una gran proporción de estudiantes negros tiene trayectorias con muchas irregularidades. Además, este trabajo muestra que el Índice de Desarrollo de la Educación Básica (IDEB) -índice oficial para medir la calidad de la educación en los municipios- puede alcanzar un valor alto a pesar de que muchos estudiantes tienen trayectorias irregulares. Esto evidenció el efecto de selectividad incorporado en este indicador. Este trabajo sugiere la incorporación de las trayectorias educativas en el indicador de seguimiento de la calidad de la educación en los municipios brasileños.

Palabras clave: Derecho a la educación. Calidad de educación. Trayectorias educativas. Datos longitudinales. Educación básica. Censo escolar. 